\title{
Ni tipo 1 ni tipo 2: una diabetes de perfil infrecuente con base autoinmunitaria
}

\begin{abstract}
Miguel Martín-Romero, Miriam Navarro-Rodríguez, José David Galián-Ramírez, Raquel Pérez-Luján, Antonia María Castillo-Navarro Hospital Clínico Universitario Virgen de la Arrixaca. Murcia. España
\end{abstract}

Recibido: 09/11/2018

Aceptado: 01/01/2019

En línea: 31/01/2019

Citar como: Martín-Romero M, Navarro-Rodríguez M, Galián-Ramírez JD, Pérez-Luján R, Castillo-Navarro AM. Ni tipo 1 ni tipo 2: una diabetes de perfil infrecuente con base autoinmunitaria. Rev Esp Casos Clin Med Intern (RECCMI). 2019 (Ene); 4(Supl 1): 14-15. doi: 10.32818/reccmi.a4s1a6.

Autor para correspondencia: Miguel Martín-Romero. miguelmr1990@gmail.com

\section{Palabras clave \\ $\triangleright$ Diabetes mellitus \\ $\triangleright$ Síndrome poliglandular autoinmune \\ $\triangleright$ Diabetes autoinmune latente del adulto}

\section{Keywords \\ $\triangleright$ Diabetes mellitus \\ $\triangleright$ Autoinmune polyglandular syndrome \\ $\triangleright$ Latent autoinmune diabetes in adults}

\begin{abstract}
Resumen
Paciente de 66 años con antecedente de enfermedad de Graves-Basedow que ingresó a cargo de Medicina Interna por diabetes mellitus de debut, siendo inicialmente catalogada como tipo 2. La mala respuesta al tratamiento con antidiabéticos orales, precisando insulinoterapia para conseguir un correcto control glucémico, junto con su patología de base, plantearon la sospecha de diabetes autoinmune latente del adulto. Ésta se confirmó con el hallazgo de autoanticuerpos específicos positivos. La asociación de estas dos patologías permite además clasificar a la paciente dentro de un grupo de trastornos endocrinos autoinmunes conocidos como síndromes poliglandulares, en este caso tipo 3.

Abstract
We present a 66-year-old patient with history of Graves-Basedow disease, who was admitted in charge of Inter-
nal Medicine with suspected debut of diabetes mellitus, being initially catalogued as type 2 . The poor response
to treatment with oral antidiabetics, requiring insulin therapy to achieve a proper glycemic control, along with
the underlying pathology, raised the suspicion of latent autoimmune diabetes in adults. This was confirmed by
the discovery of specific positive autoantibodies. The association between these two pathologies also makes it
possible to classify the patient within a group of autoimmune endocrine disorders known as type 3 polyglan-
dular syndrome.
\end{abstract}

\section{Puntos destacados}

$\triangleright$ La diabetes mellitus tipo LADA (diabetes autoinmune latente del adulto) es un grupo infrecuente pero probablemente infradiagnosticado de diabetes que se debe sospechar en aquellos casos con características mixtas entre DM1 y DM2, y confirmar mediante anticuerpos específicos.

$\triangleright$ La coexistencia con otras patologías autoinmunitarias puede, a su vez, constituir un síndrome poliglandular.

\section{Introducción}

La diabetes mellitus (DM) se clasifica de forma habitual en dos tipos principales: tipo 1 (DM1) y tipo 2 (DM2). Existen sin embargo otras formas de diabetes menos habituales con características particulares que no pueden ser catalogadas en ninguno de esos dos grupos. Dentro de estas formas infrecuentes se encuentra la conocida como diabetes autoinmune latente del adulto (en inglés, latent autoimmune diabetes of adults [LADA]), que presenta características mixtas ya que parece afectar a adultos en forma de DM2 pero con un proceso fisiopatológico que se asemeja más a la DM1, destacando la presen- cia de autoanticuerpos, la susceptibilidad genética y el descenso en cuanto a la secreción de insulina. La coexistencia en un mismo paciente de patologías autoinmunitarias previas constituye un argumento a favor de su presencia, y en caso de sospecha clínica será necesario solicitar los anticuerpos específicos para confirmar el diagnóstico.

\section{Historia clínica: antecedentes, enfermedad actual y exploración física}

Mujer de 66 años, sin reacciones adversas a fármacos conocidas, con antecedentes de hipertensión arterial en tratamiento con dos fármacos y buen control tensional, dislipemia en tratamiento con estatinas, fumadora de un paquete diario desde la juventud, enfermedad pulmonar obstructiva crónica (EPOC) en tratamiento inhalador con doble broncodilatación, y enfermedad de Graves-Basedow diagnosticada hacía 4 años. Esta última fue tratada con radioyodo y desde entonces la paciente se encontraba en tratamiento sustitutivo con levotiroxina por el hipotiroidismo resultante. Buena situación basal, 
paciente independiente para las actividades de la vida cotidiana; estado cognitivo óptimo.

Consultó en el Servicio de Urgencias por cuadro de 4-5 meses de evolución de astenia, náuseas acompañadas de vómitos ocasionales, polidipsia, poliuria y pérdida de peso de unos 10 kg. Una vez allí, se objetivó glucemia capilar 456 mg/dl. Constantes estables: tensión arterial 137/89 mmHg, frecuencia cardíaca 66 lpm saturación basal de oxígeno $94 \%$, temperatura 36,8 ㄷ․ Exploración física anodina excepto leve hipoventilación y algún crepitante seco en bases a la auscultación pulmonar. En sedimento de orina: cuerpos cetónicos +++ , glucosa +++ , resto normal. Analítica general sin alteraciones, exceptuando glucemia elevada de 421 mg/dl (valor normal: 82-115 mg/dl). Gasometría venosa: pH 7,37 (valor normal: 7,35-7,45), pCO 39 mmHg (valor normal: 32-45), $\mathrm{HCO}_{3} 21,7 \mathrm{mmol} / \mathrm{l}$ (valor normal: 21-28 mmol/l). Se decidió ingreso hospitalario con diagnóstico de síndrome hiperosmolar hiperglucémico y DM2 de debut. Sin embargo, al día siguiente la paciente solicitó el alta voluntaria que finalmente se le entregó y se indicó inicio de tratamiento con metformina 850 mg/12 h y glimepirida 1 mg/24 horas.

Al cabo de una semana, la paciente acudió nuevamente a Urgencias refiriendo síntomas casi idénticos, con aumento de náuseas y vómitos, objetivándose glucemia capilar 641 mg/dl. Los hallazgos analíticos fueron similares a los previamente mencionados, exceptuando leve empeoramiento de función renal de origen prerrenal (muy escasa tolerancia oral secundaria a las náuseas y vómitos). Se cursó de nuevo ingreso a cargo de Medicina Interna con el mismo diagnóstico de presunción.

\section{Pruebas complementarias}

Una vez en planta de hospitalización, se suspendieron los antidiabéticos orales, iniciando en su lugar insulinoterapia en régimen bolo-basal: análogos de insulina de acción ultrarrápida en cada comida (insulina glulisina: 6 UI8 UI-6 UI) y de acción lenta (insulina glargina: 24 UI por la mañana). Con ello se consiguió normalización de las cifras de glucemia de forma progresiva. Además, en analítica general se objetivó HbA1c 10,4\% (valor normal: 4,5-6,5\%) Dado el antecedente de enfermedad tiroidea autoinmunitaria que presentaba la paciente, la enfermedad de Graves-Basedow, se solicitó estudio inmunológico, obteniendo los siguientes hallazgos: anticuerpos anti-IA-2 positivos a título elevado (> 300,0 Ul/ml; límite superior 10,0 Ul/ml), anti-GAD positivos a título elevado (> 160,0 UI/ml; límite superior 5,0 UI/ml) y anticuerpos antiinsulina (> 40,5 $\mathrm{Ul} / \mathrm{ml}$ para un límite superior de $10 \mathrm{Ul} / \mathrm{ml}$ ). No se solicitaron nuevos anticuerpos relacionados con patología tiroidea autoinmunitaria ya que dicho estudio ya se había realizado previamente cuando fue diagnosticada la enfermedad de Graves-Basedow; además, las hormonas tiroideas extraídas durante el ingreso se encontraban en rango de normalidad, manteniéndose tratamiento con levotiroxina a su misma dosis habitual.

\section{Evolución}

Con los hallazgos previamente mencionados, se confirmó el diagnóstico de LADA. Además, el antecedente de enfermedad de Graves-Basedow diagnosti- cado hacía 4 años junto con la ausencia de insuficiencia suprarrenal permitió catalogar a la paciente dentro del grupo de patologías autoinmunitarias conocido como síndrome poliglandular tipo 3. Mediante tratamiento insulínico se consiguió mejoría clínica progresiva y desaparición de los síntomas cardinales, pudiendo ser alta a los pocos días con seguimiento posterior en consultas externas de Medicina Interna.

\section{Diagnóstico}

- Diabetes autoinmune latente del adulto (LADA).

- Síndrome poliglandular tipo 3.

\section{Discusión}

La DM tipo LADA es una patología que se incluye dentro del espectro de enfermedades con base autoinmunitaria, presentando características mixtas entre DM1 y DM2 ${ }^{1}$. Debe sospecharse en aquellos pacientes que debuten con características más propias de DM2 pero a su vez con algunos rasgos atípicos. Las principales diferencias son: edad de inicio por debajo de los 50 años, síntomas agudos en el momento del debut, IMC $<25 \mathrm{~kg} / \mathrm{m}^{2}$ e historia personal o familiar de enfermedad autoinmunitaria ${ }^{2}$. La presencia de dos o más de estos criterios tiene un 90\% de sensibilidad y un $71 \%$ de especificidad para la identificación de pacientes con LADA. El diagnóstico de certeza se debe realizar a través de la detección de anticuerpos específicos, siendo el anti-GAD el más prevalente ${ }^{3}$. En cuanto al tratamiento, las glitazonas en fases iniciales $y$, sobre todo, la insulinoterapia constituyen el tratamiento de elección, empleando incluso un esquema con insulina bolo-basal desde el inicio en aquellos casos con mal control metabólico. Además, al igual que ocurre con la DM1, la DM tipo LADA se asocia a otras patologías con base autoinmunitaria, siendo la tiroiditis la más frecuente en prácticamente todas las series. En el caso presentado existía asociación entre enfermedad autoinmunitaria tiroidea (Graves-Basedow) y otra patología de origen autoinmune, sin insuficiencia suprarrenal primaria, lo que en conjunto constituye un síndrome poliglandular tipo $3^{4}$.

\section{Bibliografía}

1. American Diabetes Association. Diagnosis and classification of diabetes mellitus. Diabetes Care. 2014 Jan; 37 Suppl 1: S81-90. doi: 10.2337/dc14S081.

2. Lutgens MW, Meijer M, Peeters B, Poulsen ML, Rutten MJ, Bots ML, et al. Easily obtainable clinical features increase the diagnostic accuracy for latent autoimmune diabetes in adults: an evidence-based report. Prim Care Diabetes. 2008 Dec; 2(4): 207-211. doi: 10.1016/j.pcd.2008.08.003. Epub 2008 Sep 23.

3. Hernández M, Mauricio D. Latent autoimmune diabetes in adults: are we paying sufficient attention? Endocrinol Nutr. 2015 Jan; 62(1): 1-3. doi: 10.1016/j.endonu.2014.12.001.

4. Rodríguez FJ, Pérez RB, Martell JAO, Takane EO. Síndrome poliglandular autoinmune tipo III. Honduras Pediatrica. 1997; 18(2): 44-46. 